\title{
Needlestick injury in a pregnant inpatient in an overcrowded hospital
}

N C Ngene, ${ }^{1,2}$ MBBS, Dip Obst (SA), Dip HIV Man (SA), MMed (Fam Med), FCOG (SA), MMed (O\&G); C O Onyia, ${ }^{1}$ MBBS;

J Moodley, ${ }^{3}$ MB ChB, FCOG (SA), FRCOG, MD; M J Titus, ${ }^{1,2}$ MB ChB, FCOG (SA), LLM, PGDip (Int Res Ethics)

${ }^{1}$ Department of Obstetrics and Gynaecology, Pietermaritzburg Metropolitan Hospitals Complex, KwaZulu-Natal, South Africa

${ }^{2}$ Department of Obstetrics and Gynaecology, Nelson R Mandela School of Medicine, University of KwaZulu-Natal, Durban, South Africa

${ }^{3}$ Women's Health and HIV Research Group, Nelson R Mandela School of Medicine, University of KwaZulu-Natal, Durban, South Africa

Corresponding author: N C Ngene (ngenenc@gmail.com)

Needlestick injury (NSI) is commonly reported among healthcare workers, but is not well documented in patients. We report a case of an NSI in an HIV-negative, gestational hypertensive patient admitted to a hospital for induction of labour at term. Owing to an insufficient number of hospital beds, patients were seated in an overcrowded corridor of the antenatal ward where a patient stepped on the needle of an inadvertently disconnected intravenous infusion set of another pregnant patient, who was HIV-infected. The injury occurred prior to labour induction. Antiretroviral post-exposure prophylaxis to prevent HIV infection was administered to the injured patient and her newborn. This report illustrates how hospital bed shortage may compromise patient safety and discusses measures to prevent NSI among patients and hospital overcrowding. 


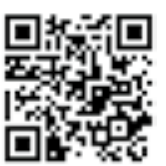

Despite the availability of medical safety programmes, ${ }^{[1]}$ more than $10 \%$ of patients suffer harm during hospital care. ${ }^{[2]}$ The occurrence of needlestick injury (NSI) in an inpatient in particular raises serious safety concern. There are reports of NSIs sustained by healthcare workers (HCWs), ${ }^{[3-5]}$ but there is no publication, to our knowledge, on such injuries occurring among inpatients. We discuss an NSI in a pregnant inpatient and outline appropriate measures to prevent such incidents.

\section{Case report}

A 25-year-old primigravida at term, who had gestational hypertension, was referred to hospital for further care. On arrival at the hospital her blood pressure was 143/93 $\mathrm{mmHg}$ and she was admitted to the antenatal ward for work-up and labour induction. Her antenatal care was uneventful prior to developing hypertension. Her risk factors for this condition were primiparity and obesity (body mass index of $30 \mathrm{~kg} / \mathrm{m}^{2}$ at booking). The clinical work-up showed no evidence of proteinuria, target organ damage or intrauterine growth restriction. During the first two days of her hospital admission, all the available beds in the antenatal ward were occupied, as the number of patients was twice the number of beds. As a result, the patient had to wait in the corridor of the antenatal ward until an empty bed became available. In this article she is referred to as the injured patient.

Another patient, referred to as the source patient, was seated on a chair in the corridor of the antenatal ward and was receiving intravenous fluids through infusion set $\mathrm{A}$ (Fig. 1). To this was attached a second intravenous infusion set, set $B$, through a needle at site $\mathrm{X}$ on set $\mathrm{A}$, to administer medication. The needle of set $\mathrm{B}$ became disconnected without being discovered by the source patient or the nurses. The source patient had been diagnosed HIV-positive 3 years previously and was on antiretroviral therapy (ART) for maternal health. The injured patient, having waited for approximately 48 hours without a bed, took to walking during the day and on her way to join other patients seated on chairs in the corridor of the antenatal ward, stepped on the disconnected needle of set $\mathrm{B}$ and sustained an NSI on her right big toe.

The patient reported the adverse event to the medical staff on duty and the following immediate measures were taken: blood was squeezed from the injured site; and the site was washed liberally with water and cleaned

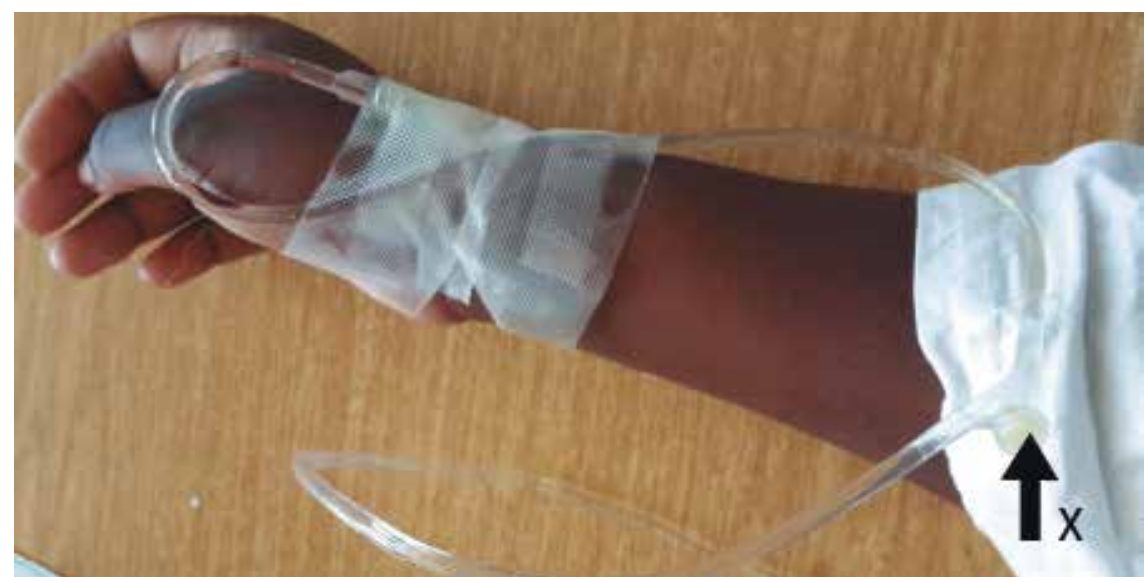

Fig. 1. Intravenous infusion set in use, showing an injection site for administration of supplementary medication. ( $X=$ injection site where an additional intravenous infusion set was connected.)

with an antiseptic solution. The patient was counselled and tested HIV-negative. She was immediately initiated on a 4 -week course of prophylactic ART as per hospital policy. Screening for hepatitis in both patients was negative, and a full blood count and liver and renal function tests of the injured patient were normal. Following hospital discharge of other stable pregnant women, the source and the injured patients were provided with beds for their continued medical care. A day after the incident, the injured patient was started on misoprostol to induce labour, but had a caesarean delivery due to fetal compromise and delivered a normal healthy boy (weight $2870 \mathrm{~g}$ ) who was started on a 6-week course of nevirapine syrup, $15 \mathrm{mg}$ daily, as recommended by the neonatologist.

The injured patient and her baby were discharged in a stable condition on the $3 \mathrm{rd}$ day after delivery. They completed the ART prophylaxis without any side-effects. Six weeks after the incident, they remained healthy, tested HIV-negative and were scheduled for further HIV testing at 3 and 6 months.

\section{Discussion}

When the optimal patient care capacity of a healthcare system or provider is exceeded, patient safety is compromised, ${ }^{[6]}$ as was seen in this case. At the time of the NSI, the number of patients in the ward was more than the available beds and the nurses who were on duty during the incident had to perform menial functions that prevented them from monitoring patients closely. The lighting was good where the injury occurred and the injured patient had no visual or physical impairment, but she may have been tired, given that she had no bed at the time of the injury.

The health facility where this incident occurred had a policy on NSI for HCWs, but owing to the rarity of NSI in patients, no policy was available for this scenario. The hospital management were aware that the number of patients in the antenatal ward usually exceeds the available beds, and had a long-term plan to construct additional wards to prevent overcrowding. Nonetheless, the adverse incident was reported to the hospital management and the interim actions taken included starting the process of converting sections of other less busy wards with extra beds to antenatal ward extensions, and deployment of additional staff to the antenatal ward.

To our knowledge, this is the first reported case where: (i) a pregnant inpatient had an NSI; and (ii) nevirapine was administered to a baby due to an NSI sustained by the mother. Nevirapine was used as it is recommended for prevention of mother-to-child transmission (PMTCT) of HIV in South Africa. ${ }^{[7]}$ Owing to the urgent need to avert further NSIs in patients, particularly in overcrowded health facilities, we outline preventive measures to be taken before the injured patient and her baby complete their follow-up visits (Table 1). These consist of components relating to: health facility administration, including developing a patient NSI policy; HCWs, including safe use of needle-containing devices; and patients and their visitors adhering to the patients' rights charter and hospital policies. These interventions are based on the authors' many years of clinical experiences in different countries. Nonetheless, different settings may require other interventions and the personnel responsible for implementing a particular task may vary.

\section{Conclusion}

NSI in inpatients has notbeen reported previously. The lesson is that it can occur, although it is preventable using the suggested measures. 
Table 1. Measures to prevent NSI in patients at different levels of the healthcare system

\section{Administration}

Develop a policy on NSI sustained by patients

Organise continuing medical education on NSI for staff and medical trainees

Ensure good record keeping so that quality data are available for audit

Organise quality improvement projects on patient safety and NSI

Ensure that patient safety is addressed as part of HCW training

Ensure that staff are aware of what to do in the event of an NSI

Improve structural facilities of the health institution as the need arises

Provide good lighting in the hospital environment

Ensure a regular supply of clean water to the health facility

Provide appropriate equipment for patient care

To address patient overcrowding of a health facility:

provide additional space where patients can be cared for; support referral of patients to alternative facilities; redeploy additional staff to work in the overcrowded unit; educate patients; support the establishment of a primary healthcare centre within or near the health facility for management of low-risk patients; if possible ensure that patients do not share one bed; inform district/ municipal authority in charge of overcrowding should it persist despite appropriate measures having been taken, etc.

\section{Provide appropriate staffing}

Provide clerks, porters and phlebotomists in the wards so that nurses/doctors can concentrate on their duties

Provide social workers, clinical psychologists, infectious disease physicians, occupational health personnel and appropriate medications to aid in managing any patient who sustains an NSI

Promote the treatment and prevention of HIV infection

Promote vaccination against hepatitis

Provide good leadership and staff motivation

Promote teamwork among staff in the health facility

Promote an awareness of patients' rights charter

Regulate visiting hours in the health facility

Provide appropriate laboratory facilities

Establish appropriate referral routes

\section{HCWs}

Adhere to guidelines on NSIs

Educate patients on what to do if there is an NSI

Show empathy, support and provide medical care to any patient who sustains an NSI

Report NSIs

Adhere to treatment protocols and consult senior colleagues for advice if necessary

Manage patients at risk of injury (such as children, the mentally ill, the blind and unconscious patients, etc.) appropriately

Counsel patients before any medical procedure

To address patient overcrowding of a health facility:

consider discharging stable patients; inform the supervisor;

discuss with management, etc.

\section{Avoid inappropriate prescription of parenteral therapies}

Use needle-free devices where possible, e.g. needle-free syringes and intravenous infusion sets

Ensure that all intravenous fluid administration sets are properly fastened to prevent inadvertent disconnection

Monitor patients with intravenous/intra-arterial lines

Remove intravenous access as soon as there is no indication for their use

Adhere to standard operating procedures regarding the safe use/ sterilisation of equipment and waste disposal

Avoid unnecessary admission of patients for inhospital care

Engage in community health promotion so as to prevent illnesses

Patients and their visitors

Ask questions when in doubt

Adhere to medical advice

Adhere to health policies such as appropriate referral routes and

facility visiting hours

NSI = needlestick injury; $\mathrm{HCW}=$ healthcare worker.

\section{References}

1. World Health Organization. WHO Patient Safety - Programme Areas. http://www. who.int/patientsafety/about/programmes/en/ (accessed 6 March 2014).

2. World Health Organization. 10 Facts on Patient Safety. http://www.who.int/ features/factfiles/patient_safety/patient_safety_facts/en/index.html (accessed 6 March 2014)

3. Gounden YP, Moodley J. Exposure to human immunodeficiency virus among healthcare workers in South Africa. Int J Gynaecol Obstet 2000;69(3):265-270. [http://dx.doi.org/10.1016/S0020-7292(00)00207-1]

4. US Public Health Service Working Group. Updated US Public Health Service Guidelines for the Management of Occupational Exposures to HIV and
Report concerns to HCWs (and to management if not satisfied) Be aware of patient and visitor rights
Recommendations for Postexposure Prophylaxis. http://stacks.cdc.gov/view/ cdc/20711 (accessed 6 March 2014).

5. Hoffmann C, Buchholz L, Schnitzler P. Reduction of needlestick injuries in healthcare personnel at a university hospital using safety devices. J Occup Med Toxicol 2013;8:1-5. [http://dx.doi.org/10.1186/1745-6673-8-20]

6. Jha AK, Prasopa-Plaizier N, Larizgoitia I, Bates DW. Patient safety research: An overview of the global evidence. Qual Saf Health Care 2010;19(1):42-47. [http:// dx.doi.org/10.1136/qshc.2008.029165]

7. South African National Department of Health. The South African Antiretroviral Treatment Guidelines 2013. http://web.up.ac.za/sitefiles/file/45/1335/877/ PMTCT\%20guidelines_March\%202013_DoH.pdf (accessed 6 March 2014). 